\title{
Cost-Effectiveness of iGlarLixi Versus iDegLira in Type 2 Diabetes Mellitus Inadequately Controlled by GLP-1 Receptor Agonists and Oral Antihyperglycemic Therapy
}

\author{
Rory J. McCrimmon · Mark Lamotte · Mafalda Ramos • \\ Abdul Jabbar Omar Alsaleh · Elisabeth Souhami · Elisheva Lew
}

Received: August 4, 2021 / Accepted: September 14, 2021 / Published online: October 29, 2021

(C) The Author(s) 2021

\section{ABSTRACT}

Introduction: The fixed-ratio combinations (FRCs) of glucagon-like peptide 1 receptor agonists (GLP-1 RAs) and basal insulin, insulin glargine $100 \mathrm{U} / \mathrm{mL}$ plus lixisenatide (iGlarLixi), and insulin degludec plus liraglutide (iDegLira), have demonstrated safety and efficacy in patients with type 2 diabetes mellitus (T2DM) inadequately controlled on GLP-1 RAs. However, a comparative cost-effectiveness analysis between these FRCs from a UK Health Service perspective has not been conducted.

Methods: The IQVIA Core Diabetes Model was used to estimate lifetime costs and outcomes in

Supplementary Information The online version contains supplementary material available at https:// doi.org/10.1007/s13300-021-01156-1.

R. J. McCrimmon

School of Medicine, University of Dundee, Dundee, UK

M. Lamotte

IQVIA Global HEOR, Zaventem, Belgium

M. Ramos

IQVIA Global HEOR, Porto Salvo, Portugal

A. J. O. Alsaleh

Department of Economics, University of Bologna,

Bologna, Italy

E. Souhami · E. Lew $(\bowtie)$

Sanofi, Paris, France

e-mail: Elisheva.Lew@sanofi.com patients with T2DM receiving iGlarLixi (based on the LixiLan-G trial) versus iDegLira (based on relative treatment effects from an indirect treatment comparison using data from DUAL III). Utilities, medical costs, and costs of diabetes-related complications were derived from literature. Model outputs included costs and quality-adjusted life years (QALYs). Incremental cost-effectiveness ratios were calculated with a local willingness-to-pay threshold of $£ 20,000$ per QALY. Extensive scenario, one-way sensitivity, and probabilistic sensitivity analyses were conducted to evaluate the robustness of the model.

Results: iGlarLixi was less costly (iGlarLixi, $£ 30,011$; iDegLira, $£ 40,742$ ), owing to lower acquisition costs, and similar in terms of QALYs gained (iGlarLixi, 8.437; iDegLira, 8.422). Extensive scenario and sensitivity analyses supported the base case findings.

Conclusion: In patients with T2DM and inadequate glycemic control despite GLP-1 RAs, use of iGlarLixi was associated with substantial cost savings and comparable utility outcomes. iGlarLixi can be considered as cost-effective versus iDegLira from the UK Health Service perspective.

Keywords: Cost-effectiveness; Cost-utility; GLP-1 receptor agonist; iGlarLixi; Type 2 diabetes mellitus; United Kingdom 


\section{Key Summary Points}

Individuals with type 2 diabetes mellitus (T2DM) inadequately controlled on glucagon-like peptide 1 receptor agonists (GLP-1 RAs) often require treatment intensification with dual or triple therapy.

To facilitate co-administration of more than one antidiabetic therapy, fixed-ratio combination (FRC) of insulin glargine $100 \mathrm{U} / \mathrm{mL}$ (iGlar) plus lixisenatide (iGlarLixi; Suliqua $\left.{ }^{\circledR}\right)$, and insulin degludec plus liraglutide (iDegLira; Xultophy ${ }^{\circledR)}$ have been developed and have demonstrated efficacy compared with continuation of GLP-1 RAs in individuals with T2DM.

This cost-effectiveness analysis compared these FRCs in patients inadequately controlled on GLP1-RAs from a UK Health Service perspective

iGlarLixi was less costly (iGlarLixi, $£ 30,011$; iDegLira, $£ 40,742$ ) owing to lower acquisition costs, and similar in terms of QALYs gained (iGlarLixi, 8.437; iDegLira, 8.422), which was supported by results of scenario and sensitivity analyses.

Thus, in patients with T2DM and suboptimal glycemic control on GLP-1 RA therapy, iGlarLixi was associated with substantial cost savings and comparable utility outcomes compared with iDegLira, and was considered cost-effective over a lifetime time horizon

\section{INTRODUCTION}

Guidelines from the American Diabetes Association (ADA) and the European Association for the Study of Diabetes (EASD) recommend glucagon-like peptide 1 receptor agonists (GLP1 RAs) as first injectable medications for individuals with glycated hemoglobin (HbA1c) above their individual targets [1, 2]. In 2019, the ADA/EASD updated their guidance to also recommend GLP-1 RA for individuals with type 2 diabetes mellitus (T2DM) at high risk of cardiovascular disease, independent of their glycemic control, in order to reduce the risk for major cardiovascular events [1]. Despite clear evidence for the efficacy of GLP-1 RA, a significant proportion of patients receiving GLP-1 RA do not experience lasting glycemic control and require treatment intensification $[3,4]$.

To facilitate co-administration of more than one antidiabetic therapy, fixed-ratio combination (FRC) products have been developed with the aim of simplifying treatment regimens compared with co-administration of individual therapies $[5,6]$. As such, FRCs of insulin glargine $100 \mathrm{U} / \mathrm{mL}$ (iGlar) plus lixisenatide (iGlarLixi; Suliqua $\left.{ }^{\circledR}\right)$, and insulin degludec plus liraglutide (iDegLira; Xultophy $\left.{ }^{\circledR}\right)$ have been developed and have demonstrated proven efficacy compared with continuation of GLP-1 RAs in patients inadequately controlled on GLP1 RAs and other oral antidiabetic drugs (OADs) $[7,8]$. The randomized, open-label LixiLan-G trial (NCT02787551) demonstrated that switching to iGlarLixi improved glucose control compared with continuing treatment with once- or twice-daily or once-weekly GLP-1 RA, in patients with T2DM insufficiently controlled on a maximum tolerated dose of GLP-1 RA plus metformin and other OADs [7]. The open-label DUAL III trial (NCT01676116), evaluating iDegLira versus continued use of previous liraglutide or exenatide therapy in patients with T2DM insufficiently controlled on a maximum tolerated dose of GLP-1 RA, demonstrated improved glycemic control compared with unchanged GLP-1 RA [8].

Although a direct head-to-head comparison of different FRCs in patients with inadequate glycemic control while receiving GLP-1 RA is lacking, an indirect treatment comparison (ITC) reported no significant differences in HbA1c target attainment, pre-prandial, or postprandial self-monitored plasma glucose for patients using either iGlarLixi or iDegLira [9]. The mean differences (95\% CI) in blood glucose parameters between iDegLira and iGlarLixi were $0.36 \% \quad(-0.58$ to -0.14$)$ for $\mathrm{HbA1c}$ 
and $-1.0 \mathrm{mmol} / \mathrm{L}(-1.57$ to -0.43$)$ for fasting plasma glucose, favoring iDegLira, at week 26 [9]. In both primary trials an increase in body weight was noted in the FRC group relative to the comparator arm, which continued with GLP-1 RA therapy [7, 8]; however, body weight change was not significantly different between iDegLira and iGlarLixi at week 26 in the ITC $(-0.23 \mathrm{~kg}$ [ -1.14 to 0.67]) [9]. Formal comparisons of hypoglycemia were unfortunately limited by differences in definitions between the two studies. To address increasing pressure on heathcare system expenditures, healthcare value has to be considered as part of decision-making. Economic evaluations are critical for decision-making processes and maximizing resource allocation. The present study sought to compare the cost-effectiveness of FRC iGlarLixi with FRC iDegLira, using the IQVIA Core Diabetes Model (CDM).

This article is based on previously conducted studies and does not contain any new studies with human participants or animals performed by any of the authors.

\section{METHODS}

\section{Study Overview}

The clinical setting for this model was patients with T2DM who have inadequately controlled HbA1c while on GLP-1 RA and OADs, in line with the LixiLan-G trial cohort [7]. Patients were entered into the model with $\mathrm{HbA1c}>$ $7.8 \%$, and with baseline characteristics based on a weighted average from the LixiLan-G clinical trial [7] (see the Electronic Supplementary Material [ESM] Table S1). Treatment effects from LixiLan-G were used to model initial responses to iGlarLixi therapy. Relative treatment effect for iDegLira was based on a previously published ITC comparing iGlarLixi and iDegLira (Table 1) [9]. Long-term clinical and cost outcomes were predicted by the IQVIA CDM version 9.5. The CDM is a validated, nonproduct-specific analysis tool that models the effect of glucose monitoring, diabetes therapies, screening, and treatment strategies for microvascular complications, treatment
Table 1 Treatment effects during initial 26 weeks with iGlarLixi and iDegLira

\begin{tabular}{lll}
\hline $\begin{array}{l}\text { LSM change } \\
\text { from baseline }\end{array}$ & $\begin{array}{l}\text { iGlarLixi } \\
\text { (based on } \\
\text { LixiLan-G) [7] }\end{array}$ & $\begin{array}{l}\text { iDegLira (based } \\
\text { on ITC by Home } \\
\text { 2020) [9] }\end{array}$ \\
\hline HbAlc, \% (SD) & $-1.02(0.05)$ & $-1.38(0.10)$ \\
$\mathrm{BMI}^{\mathrm{a}}, \mathrm{kg} / \mathrm{m}^{2}$ & 0.67 & 0.67 \\
$\begin{array}{l}\text { Symptomatic } \\
\text { hypoglycemia, } \\
\text { events/100 PY }\end{array}$ & 25 & $175^{\mathrm{c}}$ \\
$\begin{array}{l}\text { Severe } \\
\text { hypoglycemia } \\
\text { type 2, events/ }\end{array}$ & \\
100 PY & 0 \\
\hline
\end{tabular}

$B M I$ body mass index, $H b A l c$ glycated hemoglobin, iDegLira insulin degludec plus liraglutide, iGlarLixi insulin glargine $100 \mathrm{U} / \mathrm{mL}$ plus lixisenatide, ITC indirect treatment comparison, $L S M$ least squares mean, $P Y$ patientyear, $S D$ standard deviation

${ }^{a}$ BMI change from baseline was estimated from body weight change from baseline

b Defined as symptomatic with plasma glucose $<54 \mathrm{mg}$ / $\mathrm{dL} \quad(<3.0 \mathrm{mmol} / \mathrm{L})$ for iGlarLixi; $\leq 56 \mathrm{mg} / \mathrm{dL}$ $(\leq 3.1 \mathrm{mmol} / \mathrm{L})$ regardless of symptoms or severity for iDegLira

${ }^{c}$ As reported in iDegLira Dual III trial

strategies for end-stage complications, and potential intervention sequences. The IQVIA CDM has been extensively validated and is widely used in diabetes research [10-12].

Disease progression in the CDM is predicted on the basis of a series of interdependent Markov sub-models that simulate progression of disease-related complications using a set of equations for progression of the disease risk factors (United Kingdom Prospective Diabetes Study [UKPDS] Outcomes Model no. 68 UKPDS 68]) [13] and for predicting the cardiovascular and mortality risk (UKPDS 82) [14]. Each submodel uses time-, state- and diabetes type-dependent probabilities. This cost-effectiveness/cost-utility analysis was conducted from the perspective of the UK National Health Service (NHS), assuming a willingness-to-pay 
(WTP) threshold of $£ 20,000 /$ quality-adjusted life year (QALY), and used a hypothetical cohort of 1000 patients with a lifetime time horizon; 1000 model iterations were run, and annual discounting rates of $3.5 \%$ for costs and outcomes were used in line with UK National Institute of Health and Care Excellence Decision Support Unit guidance [15].

\section{Model Inputs and Structure}

The impact of iGlarLixi on risk factors (HbA1c, body mass index [BMI], symptomatic and severe hypoglycemia event rates) were applied from the LixiLan-G study (Table 1). For the comparison with iDegLira, the absolute HbA1c treatment effect was derived from the relative treatment effects reported by Home et al. [9] (Table 1). The HbA1c treatment effect of iDegLira was calculated by applying the difference obtained from ITC $(-0.36 \%$ [ -0.58 to -0.14$])$ to the treatment effect for iGlarLixi. Patients were assumed to receive either iGlarLixi or iDegLira until HbA1c progression (as simulated with the UKPDS 68 equation [13]) returned to study baseline values (i.e., 7.8\%); at this point, patients were assumed to receive treatment intensification with bolus insulin injection (rescue treatment). HbA1c reductions during rescue treatment were conservatively approximated with data from the GetGoal Duo-2 trial [16], which reported HbA1c reductions of $0.6 \%$ when an insulin bolus was added to basal insulin (with concomitant OADs). BMI was assumed to remain identical between comparator arms, and hypoglycemia rates were simulated with CDM risk equations [17].

For all simulations, QALYs were assessed using the minimum approach, where the lowest-state utility of all concurrent comorbidities was used and disutilities were added for events that occur in that year, resulting in an annual utility score for each simulated patient [12]. A comprehensive set of utility and disutility weightings were used for each model state and complication experienced, obtained from published literature (ESM Table S2) [18]. Direct medical costs during each year of therapy in the model were calculated on the basis of drug
Table 2 Annual treatment costs (GBP) in first-line and rescue therapy

$\begin{array}{ll}\text { iGlarLixi } & \text { iDegLira } \\ (\mathfrak{E}) & (\mathfrak{E})\end{array}$

First-line therapy

\begin{tabular}{lrr} 
Acquisition cost (1st year) & 1018.21 & 1667.08 \\
$\begin{array}{l}\text { Acquisition cost }(\geq 2 \mathrm{nd} \\
\text { year) }\end{array}$ & 857.97 & 1667.08 \\
Metformin add-on & 44.37 & 44.37 \\
$\begin{array}{l}\text { Administration costs } \\
\text { (needles) }\end{array}$ & 37.62 & 37.62 \\
Self-glucose monitoring & 90.55 & 90.55 \\
Annual cost (1st year) & 1190.75 & 1839.62 \\
Annual cost ( $\geq$ 2nd year) & 1030.51 & 1839.62 \\
Rescue therapy & & \\
Rapid-acting insulin & 70.04 & 70.04 \\
Additional needle use & 37.62 & 37.62 \\
First-line treatment & 939.97 & 1749.07 \\
Self-glucose monitoring & 181.09 & 181.09 \\
Annual cost & 1228.72 & 2037.83 \\
\hline
\end{tabular}

GBP British pound sterling, iDegLira insulin degludec plus liraglutide, iGlarLixi insulin glargine $100 \mathrm{U} / \mathrm{mL}$ plus lixisenatide

${ }^{a}$ All patients in iGlarLixi arm assumed to be receiving the SoloStar pen 30-60 units (delivering dose steps from 30 to 60 units of insulin glargine in combination with $10-20 \mu \mathrm{g}$ lixisenatide) from 2 nd year onwards

acquisition costs, glucose monitoring costs, management costs, and costs of T2DM complications (Table 2). In the European Union (EU), iGlarLixi is available as two FRCs: 100 units/mL insulin glargine plus $50 \mu \mathrm{g} / \mathrm{mL}$ lixisenatide (Suliqua ${ }^{\circledR}$ SoloStar pen 10-40), used to administer doses between 10 and 40 dose steps, and 100 units $/ \mathrm{mL}$ insulin glargine plus $33 \mu \mathrm{g} / \mathrm{mL}$ lixisenatide (Suliqua ${ }^{\circledR}$ SoloStar pen $30-60$ ), used to administer doses between 30 and 60 dose steps. The assumed cost of iGlarLixi in the first year was estimated on the basis of assumed use of the iGlarLixi 100/50 FRC for 3 months 
followed by use of the iGlarLixi 100/33 FRC for the remaining 9 months of that first year. From the second year onward, it was assumed that only the iGlarLixi 100/33 formulation was used. All patients were assumed to be also receiving metformin as oral diabetes therapy concurrently. A comprehensive list of costs associated with complications (including cardiovascular disease [CVD] complications, renal complications, acute events, eye disease, neuropathy, foot ulcer, and amputations) was derived from published literature, based on appropriate UK national sources [18]. These costs (in 2019, British pound sterling [GBP]) were then applied to each complication or event experienced by patients in the model (ESM Table S3; ESM Fig. S1).

\section{Analyses}

Incremental differences in costs, life years (LYs), and QALYs were obtained for iGlarLixi versus iDegLira arms. Incremental cost-effectiveness ratio (ICER) estimates for iGlarLixi versus iDegLira were calculated as the cost differential divided by the difference in QALYs and reported as costs per QALY. Cost impact was additionally assessed using the net monetary benefit (NMB). The NMB is calculated by multiplying the WTP threshold (GBP $£ 20,000 / \mathrm{QALY}$ ) by the incremental QALY and subtracts from this the incremental cost. A positive NMB value indicates that an intervention is cost-effective.

Multiple-scenario and one-way sensitivity analyses were also performed to test the robustness of the base case model assumptions. The impact of the annual HbA1c progression was tested assuming either a linear progression of 0.15 or a progression according to the Swedish National Diabetes Registry equations. The influence of the treatment effect was evaluated using upper and lower estimates of HbA1c progression in the iDegLira arm reported in the ITC (lower bound, $-0.14 \%$ [i.e., change from baseline for iDegLira, $-1.16 \%]$; upper bound, $-0.58 \%$ [i.e., change from baseline for iDegLira, - 1.60]). A similar approach using upper and lower estimates of BMI progression in the iDegLira arm (lower bound, $-1.14 \mathrm{~kg}$ less than
iGlarLixi [i.e., BMI increase for iDegLira is $0.27 \mathrm{~kg} / \mathrm{m}^{2}$ ]; upper bound, $+0.67 \mathrm{~kg}$ less than iGlarLixi [i.e., BMI increase for iDegLira is $\left.0.91 \mathrm{~kg} / \mathrm{m}^{2}\right]$ ) was tested. Finally, the cost of rescue therapy in the iGlarLixi arm was increased to match the iDegLira arm cost.

A probabilistic sensitivity analysis (PSA) was also conducted to test uncertainty in the model by random variation of key parameter inputs within plausible distributions. The parameters included in the PSA are the per-individual characteristics, treatment efficacy, utility, and cost of complications. Log-normal distributions and $20 \%$ variation were applied to sample the cost of complications. Treatment effects and utility data were sampled following the beta distribution, based on the estimated standard error detailed in Table 1 (for treatment effects) and on the standard deviation values reported by Ramos et al. [18] (for utilities) (ESM Table S2). For sampling individual baseline characteristics, truncated normal distributions were used following the mean and standard deviations reported in ESM Table S1. The PSA was performed using the base case settings and a non-parametric bootstrapping approach in which the progression of diabetes was simulated in 1000 patients, each run through the model 1000 times, to calculate the mean and standard deviation of costs, life expectancy, and qualityadjusted life expectancy. Results are presented in the cost-effectiveness plane, and as cost-effectiveness acceptability curves, giving the probability that an intervention is cost-effective for a range of WTP thresholds.

\section{RESULTS}

\section{Base Case Analysis}

In the base case analysis, the model predicted similar discounted total LYs gained in the iDegLira and iGlarLixi arms (13.177 versus 13.171, respectively; Table 3). Treatment switch to rescue therapy was predicted to occur after 4 years in the iGlarLixi arm and after 5 years in the iDegLira arm. Discounted total QALYs gained were slightly higher with iGlarLixi (iGlarLixi, 8.437; iDegLira, 8.422), driven by differences in 
Table 3 Cost-effectiveness results (base case analysis)

\begin{tabular}{lll}
\hline & iGlarLixi & iDegLira \\
\hline LY (years) & 13.171 & 13.177 \\
QALY (years) & 8.437 & 8.422 \\
Total costs $(£)$ & 30,011 & 40,742 \\
iGlarLixi versus iDegLira & & \\
Incremental LY & -0.006 & \\
Incremental QALY & 0.015 & \\
Incremental costs $(£)$ & $-10,730$ & \\
ICER per QALY $(£)$ & Dominant & \\
NMB $^{\text {a }}(£)$ & 11,030 & \\
\hline
\end{tabular}

GBP British pound sterling, ICER incremental cost-effectiveness ratio, iDegLira insulin degludec plus liraglutide, iGlarLixi insulin glargine $100 \mathrm{U} / \mathrm{mL}$ plus lixisenatide, $L Y$ life year, $N M B$ net monetary benefit, $Q A L Y$ quality-adjusted life year

a At local willingness-to-pay threshold of $£ 20,000$ per QALY

non-severe hypoglycemic events with iGlarLixi versus iDegLira (iGlarLixi, 1194 events per 1000 patient-years; iDegLira, 1201 events per 1000 patient-years; ESM Fig. S1). Costs were substantially lower with iGlarLixi, largely due to the difference in treatment acquisition costs (iGlarLixi, £16,264; iDegLira, £27,042; Table 2). In the base case analysis, iGlarLixi was less expensive and produced similar QALYs; the NMB of iGlarLixi was $£ 11,030$ (Table 3).

\section{Scenario Analyses}

The base case findings were not sensitive to multiple variations in model inputs (Fig. 1). With the exception of the scenario analysis using the lower range of BMI, iGlarLixi was less costly with similar QALYs gained versus iDegLira in all scenarios (Fig. 1). The NMB for iGlarLixi versus iDegLira ranged from $£ 10,291$ to $£ 12,476$ in most scenarios, but remained positive in all (NMB was $£ 2867$ when cost of rescue therapy was the same in each arm).

\section{Probabilistic Sensitivity Analysis}

In PSA, all iterations resulted in cost savings for iGlarLixi, and $56 \%$ of iterations were in the southeast quadrant of the cost-effectiveness plan, indicating that iGlarLixi resulted in more QALYs gained and cost savings versus iDegLira (Fig. 2a). At a WTP threshold of $£ 20,000 /$ QALY, iGlarLixi was deemed cost-effective in $100 \%$ of cases (Fig. 2b).

\section{DISCUSSION}

This analysis demonstrates that iGlarLixi is costeffective compared with iDegLira in patients with T2DM and inadequate glucose control on GLP-1 RA and OADs, both in the base case and in the multiple-scenario analyses. Life expectancy and QALYs gained over the model duration were similar between treatment arms; however, the iGlarLixi cohort had lower overall costs, largely owing to the annual acquisition costs for iGlarLixi being approximately $£ 1000$ less than iDegLira. QALYs gained were driven by differences in non-severe hypoglycemic events. Observations from the PSA further support the robustness of the calculated ICER analyses, and together suggest that iGlarLixi is a more costeffective treatment option than iDegLira for patients with T2DM requiring treatment intensification from GLP-1 RA therapy.

Despite the available evidence of efficacy and safety, economic modeling enables payers to make informed decisions regarding budgetary impact and long-term cost-effectiveness. Consequently, decision makers require a robust health-economic analysis to further inform treatment selection. Our estimation indicated that iGlarLixi offered excellent value for money, with similar QALYs and commensurate cost savings compared with iDegLira, and the costeffective acceptability curve suggested that at a WTP threshold of $£ 20,000 /$ QALY, iGlarLixi was cost-effective in $100 \%$ of cases. The value of iGlarLixi is also supported by NMB estimates, an additional measure of cost-effectiveness that indicates the estimated monetary value of the benefit of a comparative intervention [19]. In this analysis, the base case and all scenario 


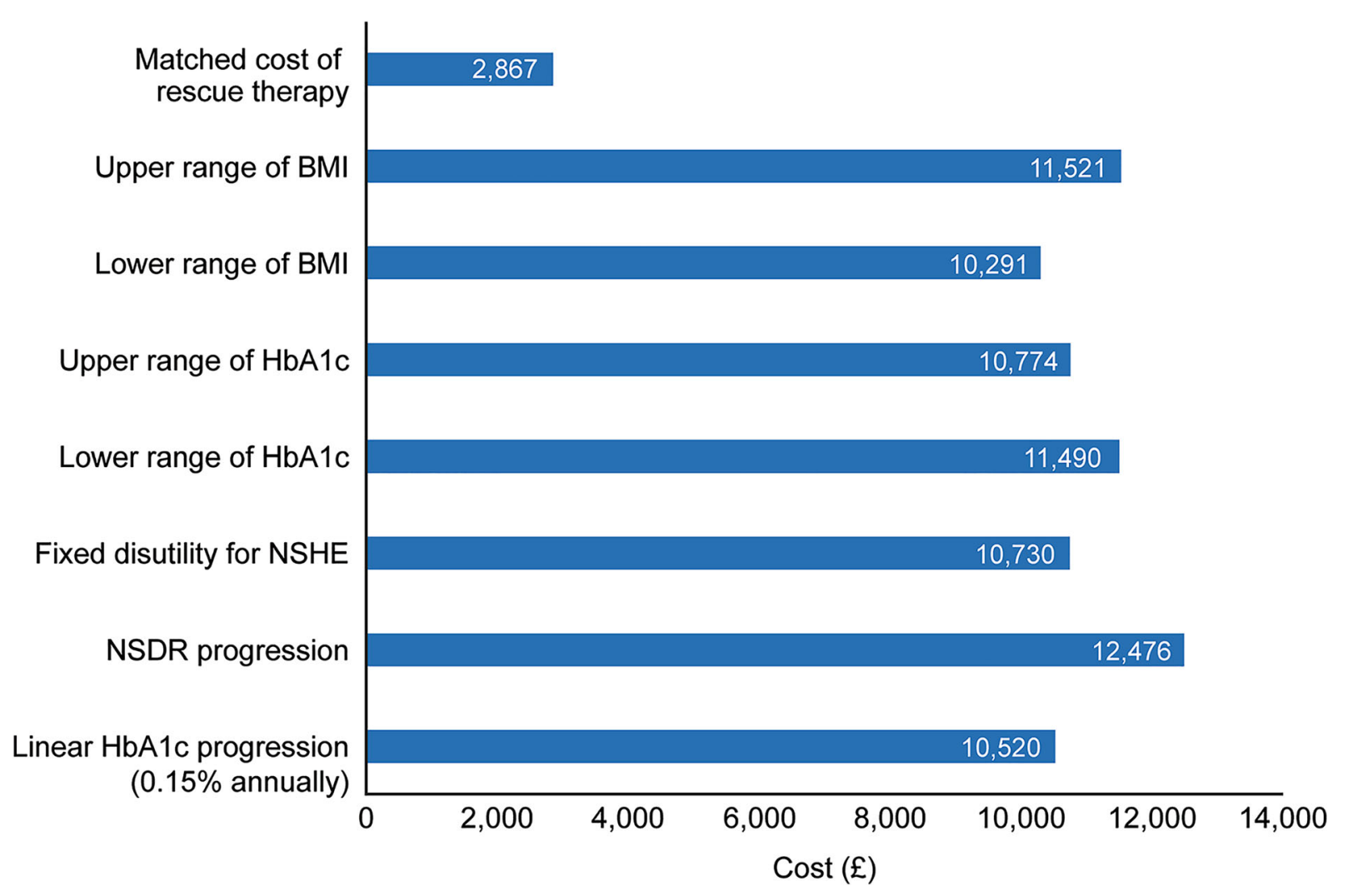

Fig. $1 \mathrm{NMB}^{\mathrm{a}}$ results for iGlarLixi versus iDegLira: summary of scenario analyses. BMI body mass index, HbAlc glycated hemoglobin, iDegLira insulin degludec plus liraglutide, iGlarLixi insulin glargine $100 \mathrm{U} / \mathrm{mL}$ plus

analyses had positive NMB estimates, indicating that the cost savings with iGlarLixi outweighed the value of the marginal differences in QALYs.

This analysis has several strengths. We assessed lifetime costs and outcomes using the IQVIA CDM, which has been extensively validated and is widely used in diabetes research [10-12]. Model inputs were drawn largely from locally appropriate published sources, and extensive scenario analyses found the model and results to be robust to variation in key assumptions. However, certain limitations must also be considered. Firstly, absolute HbA1c treatment effect for iDegLira was derived from relative $\mathrm{HbA} 1 \mathrm{c}$ treatment effects estimated from a previously published ITC [9]. As a result of differences in the threshold used to define hypoglycemia in iGlarLixi and iDegLira clinical trials $(<54 \mathrm{mg} / \mathrm{dL}$ and $\leq 56 \mathrm{mg} / \mathrm{dL}$, lixisenatide, NMB net monetary benefit, NSDR National Swedish Diabetes Registry, NSHE non-severe hypoglycemic event. ${ }^{a} \mathrm{NMB}$ calculated based on assumed local willingness-to-pay threshold of $£ 20,000$

respectively), an ITC was not conducted, and hypoglycemia event rates from the trials were used in CDM (summary reported in Home et al. [9]). However, it should be noted that these threshold definitions are similar. For BMI, there was no significant difference between iGlarLixi and iDegLira [9], so the same BMI increase is applied in both arms in the base case. Robustness of the cost-effectiveness conclusion was confirmed in all scenario and sensitivity analyses testing variations in the $\mathrm{HbA1c}, \mathrm{BMI}$, and utilities values associated with occurrence of hypoglycemia. Secondly, HbA1c reduction during rescue therapy $(-0.6 \%)$ was conservatively approximated with the basal-plus arm of the GetGoal Duo-2 trial in the absence of other evidence, and likely underestimated the treatment effect. The BMI was assumed to remain unchanged in the two treatment arms. Another 


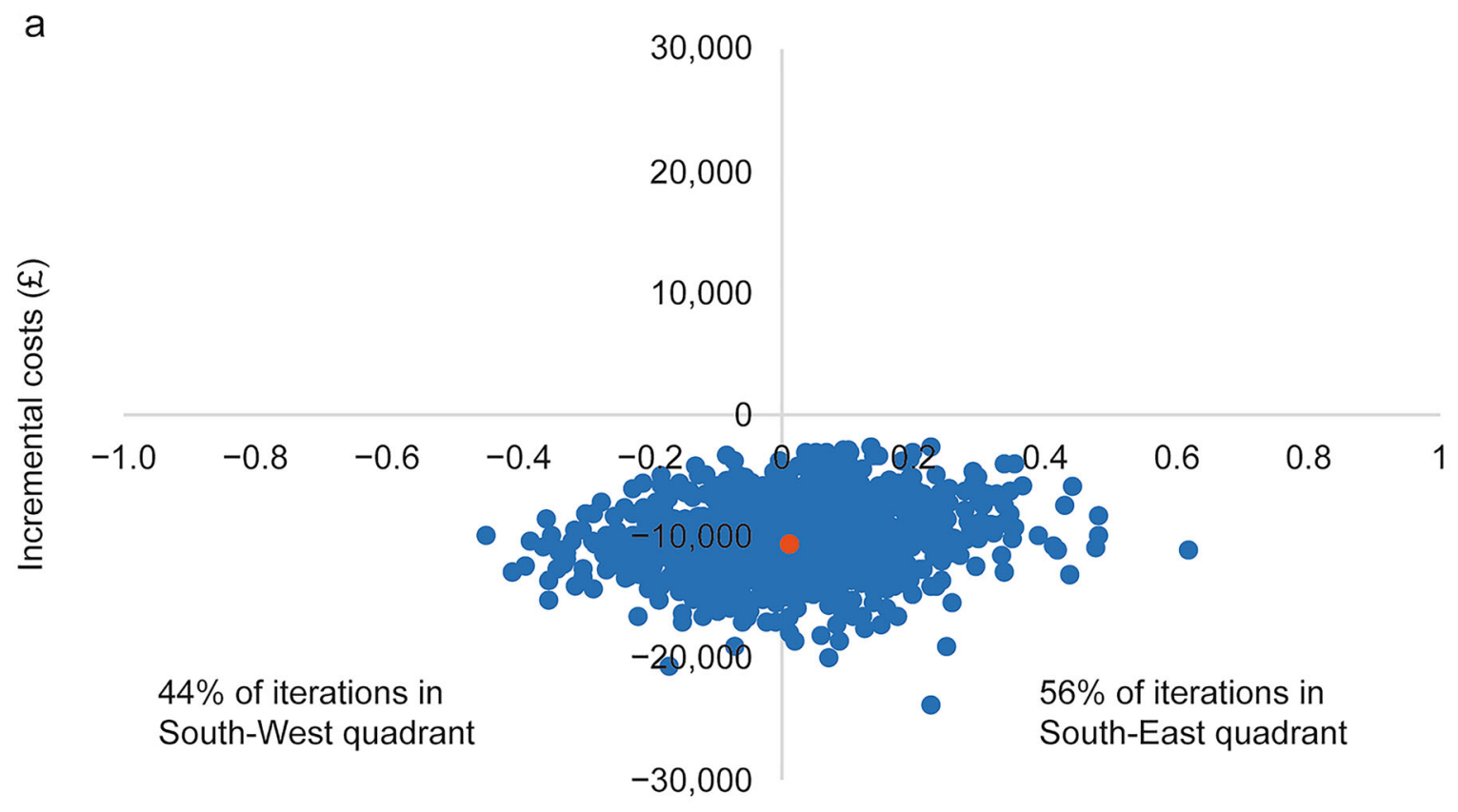

Incremental QALYS

b

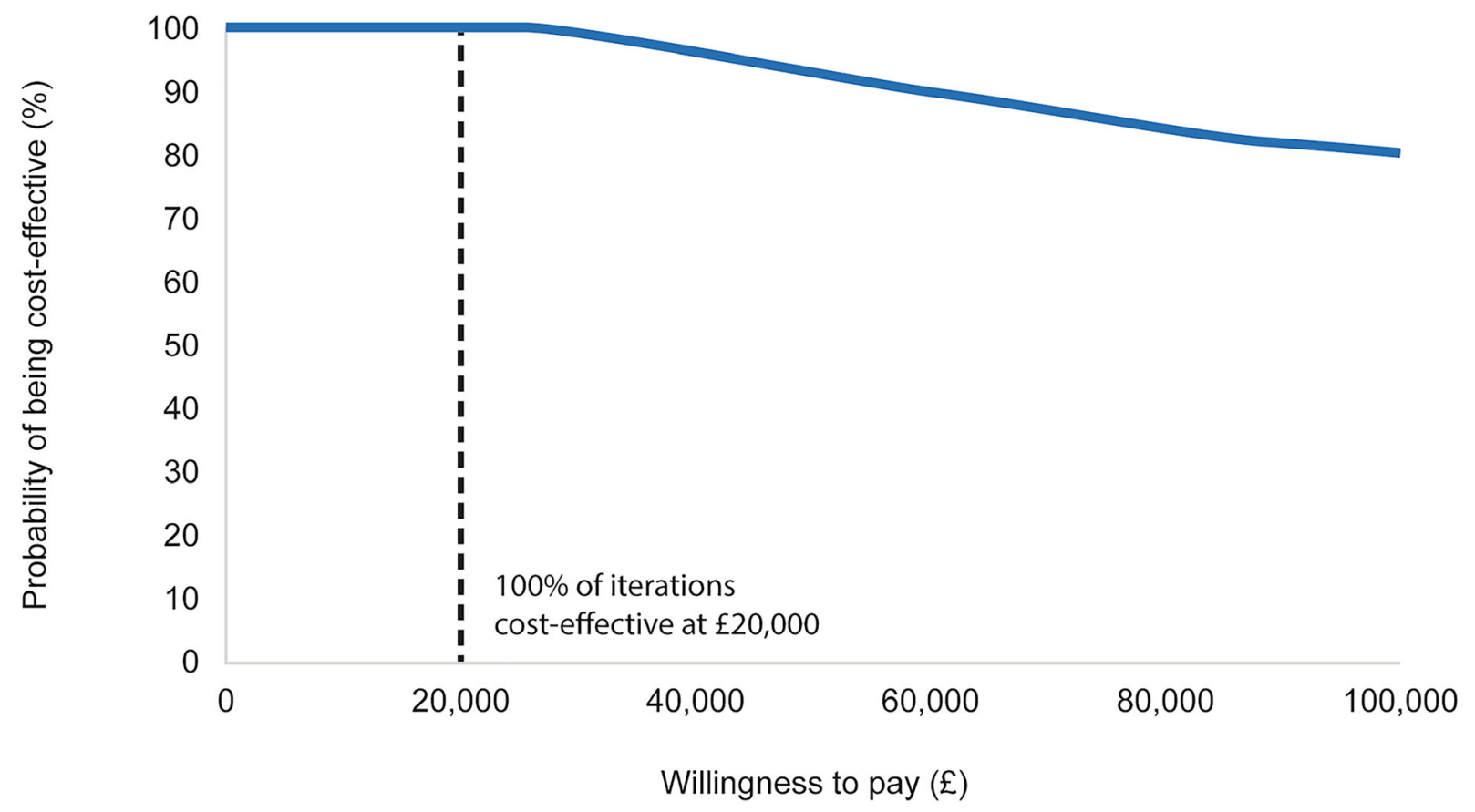

Fig. 2 Base case cost-effectiveness results: a cost-effectiveness plane; $\mathbf{b}$ cost-effectiveness acceptability curve. QALY qualityadjusted life year 
limitation was to rely on relatively short-term data (26 weeks) to extrapolate to long-term projections. This issue is common in healtheconomic analyses in diabetes but was addressed with sensitivity and scenario analyses. Furthermore, our analysis assessed only fixed-dose GLP-1 RA and insulin combinations, and did not compare free-dose combinations of the individual agents. However, this is likely to represent a cautious approach for a cost-effectiveness analysis, as free-dose combinations are known to be more expensive [20] and could be associated with poorer treatment adherence [6]; consequently, they are likely to be less cost-effective compared with the FRC products used in this analysis, although this would need to be confirmed. Cost sources used in the model were current at the time of publication, but obviously may not reflect future changes in unit cost pricing. Finally, we calculated costs in GBP as the utility analysis was conducted from the perspective of the UK NHS; extrapolation to other markets and currencies cannot be assumed.

\section{CONCLUSION}

Despite unavoidable assumptions inherent to health-economic modeling, long-term analyses are recommended by Health Technology Assessment bodies to inform the decision-making process and optimize budget allocation. In conclusion, our analyses demonstrate that iGlarLixi is a cost-effective treatment option compared with iDegLira in patients with T2DM whose HbA1c level is inadequately controlled using GLP-1 RA and other OADs, with substantial cost savings and comparable efficacy in the UK.

\section{ACKNOWLEDGEMENTS}

Funding. This study was sponsored by Sanofi. All publication costs, including the journal's Rapid Service fee, were funded by Sanofi.
Medical Writing Assistance. Medical writing support was provided by Martin Bell and Vanessa Gross of Curo (part of Envision Pharma Group) and was funded by Sanofi.

Authorship. All named authors meet the International Committee of Medical Journal Editors (ICMJE) criteria for authorship for this article, take responsibility for the integrity of the work as a whole, and have given their approval for this version to be published.

Author Contributions. All authors were responsible for the conception, study design, and to the acquisition, analysis and interpretation of data. All authors contributed substantially to drafting and revising of the manuscript. All authors have reviewed and approved the final version of the manuscript for submission to Diabetes Therapy, and agree to be accountable for its contents.

Disclosures. Rory J. McCrimmon is a member of the advisory panel for Sanofi and Novo Nordisk, a board member for NHS Tayside Health Board, an employee at University of Dundee, and has received research support from the Medical Research Council, Wellcome, EU Horizon 2020, JDRF, Diabetes UK, and Novo Nordisk. Mark Lamotte and Mafalda Ramos are employees of IQVIA, which received consulting fees for adapting the IQVIA Core Diabetes Model to allow the analyses that resulted in this manuscript. Abdul Jabbar Omar Alsaleh has received independent contractor funding from Sanofi for this analysis. Elisabeth Souhami and Elisheva Lew are employees and stockholders of Sanofi.

Compliance with Ethics Guidelines. This article is based on previously conducted studies and does not contain any new studies with human participants or animals performed by any of the authors.

Data Availability. The datasets generated during and/or analyzed during the current study are available from the corresponding author on reasonable request. 
Open Access. This article is licensed under a Creative Commons Attribution-NonCommercial 4.0 International License, which permits any non-commercial use, sharing, adaptation, distribution and reproduction in any medium or format, as long as you give appropriate credit to the original author(s) and the source, provide a link to the Creative Commons licence, and indicate if changes were made. The images or other third party material in this article are included in the article's Creative Commons licence, unless indicated otherwise in a credit line to the material. If material is not included in the article's Creative Commons licence and your intended use is not permitted by statutory regulation or exceeds the permitted use, you will need to obtain permission directly from the copyright holder. To view a copy of this licence, visit http://creativecommons.org/licenses/by$\mathrm{nc} / 4.0 /$.

\section{REFERENCES}

1. Buse JB, Wexler DJ, Tsapas A, et al. 2019 Update to: management of hyperglycemia in type 2 diabetes, 2018. A consensus report by the American Diabetes Association (ADA) and the European Association for the Study of Diabetes (EASD). Diabetes Care. 2020;43(2):487-93.

2. Davies MJ, D'Alessio DA, Fradkin J, et al. Management of hyperglycemia in type 2 diabetes, 2018. A consensus report by the American Diabetes Association (ADA) and the European Association for the Study of Diabetes (EASD). Diabetes Care. 2018;41(12):2669-701.

3. Peng XV, McCrimmon RJ, Shepherd L, et al. Glycemic control following GLP-1 RA or basal insulin initiation in real-world practice: a retrospective, observational, longitudinal cohort study. Diabetes Ther. 2020;11(11):2629-45.

4. Sikirica MV, Martin AA, Wood R, Leith A, Piercy J, Higgins V. Reasons for discontinuation of GLP1 receptor agonists: data from a real-world cross-sectional survey of physicians and their patients with type 2 diabetes. Diabetes Metab Syndr Obes. 2017;10:403-12.

5. Baumgartner A, Drame K, Geutjens S, Airaksinen M. Does the polypill improve patient adherence compared to its individual formulations? A systematic review. Pharmaceutics. 2020;12(2):190.
6. Lokhandwala T, Smith N, Sternhufvud C, Sorstadius E, Lee WC, Mukherjee J. A retrospective study of persistence, adherence, and health economic outcomes of fixed-dose combination vs. loose-dose combination of oral anti-diabetes drugs. J Med Econ. 2016;19(3):203-12.

7. Blonde L, Rosenstock J, Del Prato S, et al. Switching to iGlarLixi versus continuing daily or weekly GLP1 RA in type 2 diabetes inadequately controlled by GLP-1 RA and oral antihyperglycemic therapy: the LixiLan-G randomized clinical trial. Diabetes Care. 2019;42(11):2108-16.

8. Linjawi S, Bode BW, Chaykin LB, et al. The efficacy of IDegLira (Insulin Degludec/Liraglutide Combination) in adults with type 2 diabetes inadequately controlled with a GLP-1 receptor agonist and oral therapy: DUAL III randomized clinical trial. Diabetes Ther. 2017;8(1):101-14.

9. Home PD, Aroda VR, Blonde L, et al. Efficacy and safety of iGlarLixi versus IDegLira in adults with type 2 diabetes inadequately controlled by glucagon-like peptide-1 receptor agonists: a systematic literature review and indirect treatment comparison. Diabetes Obes Metab. 2020;22(11):2170-8.

10. Palmer AJ, Si L, Tew M, et al. Computer modeling of diabetes and its transparency: a report on the eighth mount hood challenge. Value Health. 2018;21(6):724-31.

11. Palmer AJ, Roze S, Valentine WJ, et al. The CORE diabetes model: projecting long-term clinical outcomes, costs and cost-effectiveness of interventions in diabetes mellitus (types 1 and 2) to support clinical and reimbursement decision-making. Curr Med Res Opin. 2004;20(Suppl 1):S5-26.

12. McEwan P, Foos V, Palmer JL, Lamotte M, Lloyd A, Grant D. Validation of the IMS CORE diabetes model. Value Health. 2014;17(6):714-24.

13. Clarke PM, Gray AM, Briggs A, et al. A model to estimate the lifetime health outcomes of patients with type 2 diabetes: the United Kingdom Prospective Diabetes Study (UKPDS) Outcomes Model (UKPDS no. 68). Diabetologia. 2004;47(10): 1747-59.

14. Hayes AJ, Leal J, Gray AM, Holman RR, Clarke PM. UKPDS outcomes model 2: a new version of a model to simulate lifetime health outcomes of patients with type 2 diabetes mellitus using data from the 30 year United Kingdom Prospective Diabetes Study: UKPDS 82. Diabetologia. 2013;56(9):1925-33.

15. NICE. CHTE methods review: discounting. https:// www.nice.org.uk/Media/Default/About/what-wedo/our-programmes/nice-guidance/chte-methods- 
consultation/Discounting-task-and-finish-groupreport.docx. July 2020. Accessed 31 Mar 2021

16. Rosenstock J, Guerci B, Hanefeld M, et al. Prandial options to advance basal insulin glargine therapy: testing lixisenatide plus basal insulin versus insulin glulisine either as basal-plus or basal-bolus in type 2 diabetes: the GetGoal Duo-2 trial. Diabetes Care. 2016;39(8):1318-28.

17. McEwan P, Foos V, Grant D, et al. Predicting the frequency of severe and non-severe hypoglycaemia in insulin treated type- 2 diabetes subjects. Value Health. 2013;16(7):A435.

18. Ramos M, Cummings MH, Ustyugova A, Raza SI, de Silva SU, Lamotte M. Long-term cost-effectiveness analyses of empagliflozin versus oral semaglutide, in addition to metformin, for the treatment of type 2 diabetes in the UK. Diabetes Ther. 2020;11(9):2041-55.

19. McFarlane PA, Bayoumi AM. Acceptance and rejection: cost-effectiveness and the working nephrologist. Kidney Int. 2004;66(5):1735-41.

20. Tran E. Fixed-ratio combinations. Clin Diabetes. 2017;35(4):242-6.
21. Folsom AR, Chambless LE, Duncan BB, Gilbert AC, Pankow JS. Atherosclerosis Risk in Communities Study Investigators. Prediction of coronary heart disease in middle-aged adults with diabetes. Diabetes Care. 2003;26(10):2777-84.

22. Beaudet A, Clegg J, Thuresson PO, Lloyd A, McEwan $P$. Review of utility values for economic modeling in type 2 diabetes. Value Health. 2014;17(4): 462-70.

23. Lauridsen JT, Lonborg J, Gundgaard J, Jensen HH. Diminishing marginal disutility of hypoglycaemic events: results from a time trade-off survey in five countries. Qual Life Res. 2014;23(9):2645-50.

24. Foos V, McEwan P. Conversion of hypoglycemia utility decrements from categorical units reflecting event history into event specific disutility scores applicable to diabetes decision models. Value Health. 2018;21:S223.

25. Bagust A, Beale S. Modelling EuroQol health-related utility values for diabetic complications from CODE-2 data. Health Econ. 2005;14(3):217-30. 